University of Wollongong

Research Online

Faculty of Engineering - Papers (Archive)

Faculty of Engineering and Information

Sciences

2000

\title{
A new silicon detector for microdosimetry applications in proton therapy
}

Anatoly B. Rosenfeld

University of Wollongong, anatoly@uow.edu.au

P. Bradley

University of Wollongong

I. Cornelius

University of Wollongong, iwan@uow.edu.au

G. I. Kaplan

University of Wollongong

B. J. Allen

University of Wollongong

See next page for additional authors

Follow this and additional works at: https://ro.uow.edu.au/engpapers

Part of the Engineering Commons

https://ro.uow.edu.au/engpapers/16

\section{Recommended Citation}

Rosenfeld, Anatoly B.; Bradley, P.; Cornelius, I.; Kaplan, G. I.; Allen, B. J.; Flanz, J. B.; Goitein, M.; Van Meerbeeck, A.; Schubert, J.; Bailey, J.; Takada, Y.; Maruhashi, A.; and Hayakawa, Y.: A new silicon detector for microdosimetry applications in proton therapy 2000.

https://ro.uow.edu.au/engpapers/16

Research Online is the open access institutional repository for the University of Wollongong. For further information contact the UOW Library: research-pubs@uow.edu.au 


\section{Authors}

Anatoly B. Rosenfeld, P. Bradley, I. Cornelius, G. I. Kaplan, B. J. Allen, J. B. Flanz, M. Goitein, A. Van Meerbeeck, J. Schubert, J. Bailey, Y. Takada, A. Maruhashi, and Y. Hayakawa 


\title{
A New Silicon Detector for Microdosimetry Applications in Proton Therapy
}

\author{
A.B. Rosenfeld ${ }^{1}$, P.D. Bradley ${ }^{1}$, I.Cornelius ${ }^{1}$, G.I. Kaplan ${ }^{1}$, B.J. Allen ${ }^{1}$, J.B. Flanz ${ }^{2}$, M. Goitein ${ }^{2}$, \\ A. Van Meerbeeck ${ }^{3}$, J. Schubert ${ }^{3}$, J. Bailey $^{3}$, Y. Takada ${ }^{4}$, A. Maruhashi $^{4}$, Y. Hayakawa ${ }^{4}$ \\ ${ }^{1}$ Department of Engineering Physics, University of Wollongong, NSW 2522, Australia \\ ${ }^{2}$ NPTC , Harvard Medical School, Boston MA 02114, USA \\ ${ }^{3}$ Ion Beam Applications (IBA), Belgium \\ ${ }^{4}$ University of Tsukuba, Tsukuba, Ibaraki 305, Japan
}

\begin{abstract}
A silicon-on-insulator diode array with a sensitive depth of 10 microns has been developed for microdosimetry in proton therapy. The detector was coupled to a radiation-hard charge sensitive amplifier with the probe assembly capable of measuring an LET down to $1.2 \mathrm{keV} / \mu \mathrm{m}$. The device has been successfully tested at two proton therapy centers: The 230 $\mathrm{MeV}$ Northeastern Proton Therapy Center, Boston and the $250 \mathrm{MeV}$ Proton Medical Research Center at Tsukuba, Japan. The device offers much improved spatial resolution compared with a proportional gas counter particularly in the critical high dose region around the proton Bragg peak. Due to its small cross-sectional area $\left(0.04 \mathrm{~cm}^{2}\right)$ measurements may also be made in facilities with short high intensity beams.
\end{abstract}

\section{INTRODUCTION}

Silicon detectors during the last decade have found a wide range of applications in radiation oncology. This has been greatly assisted by the development of detector systems for high energy physics (HEP) in which many advances have been made in the areas of radiation hardness and low noise multi-channel readout integrated systems.

In certain applications silicon based detectors offer significant advantages particularly with regard to spatial resolution. A new high spatial resolution silicon based microdosimeter has been developed using silicon-on-insulator (SOI) technology. Previous work characterized the sensitive volume and radiation hardness of this detector [1] and its performance in boron neutron capture therapy (BNCT) $[2,3]$.

The development of an improved low noise implementation of this device and testing at two proton therapy facilities will be discussed. Experiments were conducted at the Proton Medical Research Center (PMRC) at University of Tsukuba, KEK, Japan, using a $250 \mathrm{MeV}$ proton beam and at the Northeastern Proton Therapy Center (NPTC), Boston using a $230 \mathrm{MeV}$ proton beam.

Proton therapy is a very important radiation oncology modality for the treatment of deep-seated tumors [4]. Currently, many HEP accelerator centers involved in medical programs have dedicated beams for proton therapy and heavy ion therapy. The key advantage of proton therapy lies in the local delivery of dose at high depths in tissue due to the energy loss depth dose profile of high-energy protons in matter (Bragg peak).

Protons interact with matter by continuous slowing down as a result of Coulomb collisions with electrons and nuclei, by Bremsstrahlung radiation loss, and by nuclear reactions. The Coulomb interaction with electrons is the predominant mechanism for energy loss and is typically described by the electronic stopping power or Linear Energy Transfer (LET, $\mathrm{dE} / \mathrm{dx}$ ) of the proton [5]. Radiation loss is negligible for the energies of interest in proton therapy. However, nuclear reactions have a significant effect on the proton beam characteristics. The probability that protons will undergo a nuclear interaction whilst traversing $1 \mathrm{gm} / \mathrm{cm}^{2}$ is around $1 \%$ and after $20 \mathrm{~cm} 1$ in 4 protons will have suffered a nuclear interaction. A NIST report by Seltzer [6] and summarized by Miller [4] describes the interactions and products. Approximately $21 \%$ of the energy lost in slowing down a 250 $\mathrm{MeV}$ proton beam in water is due to nonelastic nuclear reactions. This fraction drops to around $4 \%$ for a $70 \mathrm{MeV}$ beam. Thus nuclear interactions affect the identity and energy distribution of the secondary particles and decrease the number of primary protons in the beam

The radiobiological effectiveness of the beam is dependent on the particle types and their energy distribution. An important characteristic of the beam is the LET spectrum of all primary and secondary particles responsible for the radiobiological properties of beam. Experimental microdosimetry attempts to quantify the LET spectrum by measuring the energy deposited in a cell-sized volume. This may then be used to infer radiobiological properties of the beam relevant for treatment planning. Thus, microdosimetric measurements in a water phantom are important in high energy proton therapy installations. Clearly, the relative biological effectiveness of the beam varies with depth. A 250 $\mathrm{MeV}$ proton has an initial LET of $0.4 \mathrm{keV} / \mathrm{um}$. This rises sharply in the Bragg peak region reaching a maximum value of approximately $80 \mathrm{keV} / \mu \mathrm{m}$ within a few microns of the end of the range. Microdosimetric measurements, at depths where protons enter the Bragg peak region, are particularly challenging due to the high spatial resolution required. 


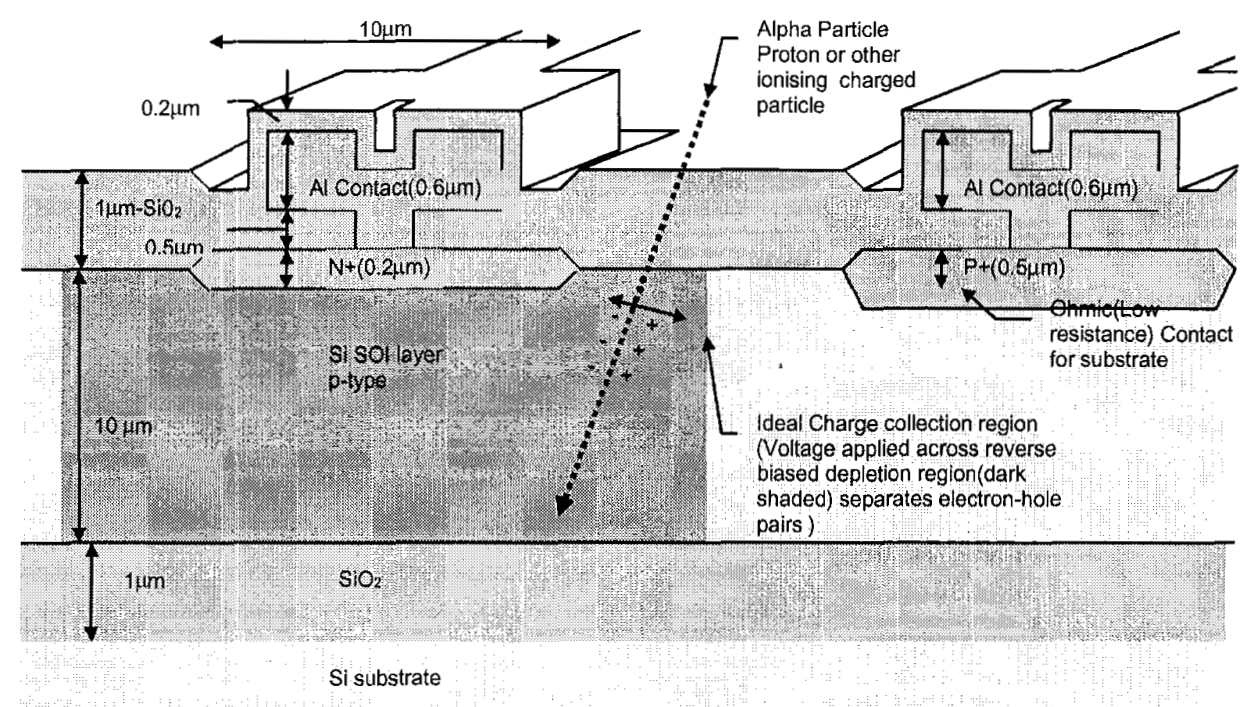

Figure 1: Detailed cross-section of diode array with approximate dimensions

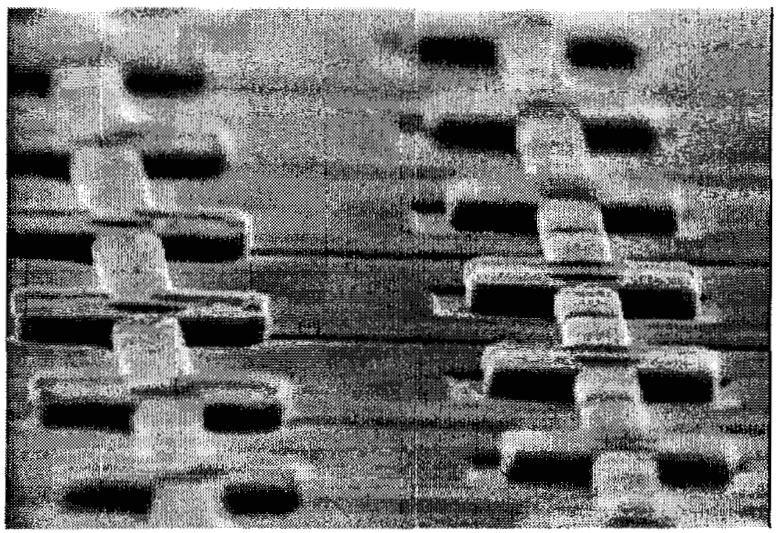

Figure 2: SEM photograph of a portion of the $10 \times 10 \mu \mathrm{m}$ diode array. (Note: Raised sections are aluminum tracks connecting contacts so that all diodes are electrically in parallel. The width of the photo is about $23 \mu \mathrm{m}$.)

Microdosimetric studies of proton beams have been performed by several investigators [7-12]. Typically, a spherical proportional counter containing a low pressure tissue equivalent gas is used to obtain the spectrum of energy deposition events [13]. The millimeter resolution required in the Bragg peak region is not achievable using such a counter which normally has a diameter of $1-2 \mathrm{~cm}$. The large size of the counter also creates problems with pileup effects due to simultaneous multiple events during high DC current operation or when the proton beam consists of large currents produced in nanosecond to microsecond pulses. Measurements of microdosimetric spectra using a proportional counter have been reported with count rates as low as 350 counts/sec still exhibiting pileup due to the large size of the counter [10]. Therefore, proton microdosimetry requires small microdosimeters with high spatial resolution $(<1 \mathrm{~mm})$ and small collection areas along with a short collection time in the tens of nanosecond range. Analysis of the pulse time structure of the beam current is important for correct measurements of microdosimetry spectra.

\section{MICRODOSIMETRY DEFINITIONS AND SPECTRUM PRESENTATION}

The energy deposited in a microdosimeter is amplified and recorded by a multi-channel analyzer to produce a spectrum of the number of events recorded at different energies. Experimental microdosimetry then introduces the concept of lineal energy $y$ (in $\mathrm{keV} / \mu \mathrm{m}$ ) which provides a crude measure of particle LET. Lineal energy is defined as

$y=\frac{\varepsilon}{\bar{l}}$

where $\varepsilon$ is the energy imparted in the volume of interest by an energy deposition event and $\bar{l}$ is the mean chord length of the volume [13]. Ionizing particles intercepting the volume will produce a range of chord lengths and $\bar{l}$ is the mean of the chord length distribution. Often $\bar{l}$ is calculated assuming the sensitive volume is immersed in a uniform isotropic fluence of radiation with infinite range [13]. Given a-priori information regarding the radiation field one may estimate a $\bar{l}$ most appropriate for the irradiation conditions. In our case, $\bar{l}=8.2 \mu \mathrm{m}$ with the estimation discussed in section III.A

Thus, the energy deposited by each event is divided by the mean chord length to give the lineal energy as per equation (1). The dose distribution is then given by

$D(y) \propto y f(y)$

where $f(y)$ is the lineal energy probability distribution calculated from the number of events in the lineal energy range interval $\mathrm{d} y$ and $D(y)$ is the dose probability density distribution [13]. Note that standard microdosimetry nomenclature presents $D(y)$ as $d(y)$ but we use the capital form to improve readability and avoid confusing $\mathrm{d} y$ and $d(y)$. The dose distribution relationship simply reflects the fact that higher lineal energies deposit a higher dose. By definition, the distribution $D(y)$ is normalized to unity: 


$$
\int_{0}^{\infty} D(y) \mathrm{d} y=1
$$

We are interested in quite a wide range of lineal energies from $1 \mathrm{keV} / \mu \mathrm{m}$ to several hundred $\mathrm{keV} / \mu \mathrm{m}$. In this range the relative biological effectiveness begins to increase above unity. Due to the wide lineal energy range, microdosimetric spectra are traditionally presented in a log-linear format, which requires further scaling to preserve the dose to area correspondence. In summary, all microdosimetric spectra are presented in the standard $y D(y) \propto y^{2} f(y)$ versus $\log (y)$ format. The ordinate is displayed as $y D(y)$ on a linear-log plot since

$$
\int D(y) \mathrm{d} y=\ln (10) \int y D(y) \mathrm{d}(\log y)
$$

and this integral is proportional to the dose deposited in the interval $\mathrm{d} y$. Presented in this way, equal areas under different regions of the function $y D(y)$ correspond to equal doses.

To convert to tissue equivalent (TE) microdosimetric spectra the $\vec{l}$ of the silicon device is simply scaled by a factor of 1/0.63. A previous paper by Bradley [14] has addressed the issue of tissue equivalency of silicon based microdosimetric measurements for BNCT $[2,3]$. The results are also applicable for energy deposition through coulomb interactions by higher energy protons. It was demonstrated [14] that under appropriate geometrical scaling (dimensions multiplied by 1/0.63) silicon detectors with well known geometry will record energy deposition spectra representative of tissue cells of equivalent shape. Note, that the study neglected nuclear reactions in the silicon since the incident charged particles, particularly protons, will be much more frequent. In this paper, with an emphasis on device operation, we present all results based on the silicon mean chord length without TE correction.

\section{METHODS}

\section{A. New Silicon Microdosimeter}

The silicon microdosimeter consists of a SOI diode array test structure fabricated by Fujitsu Research Laboratories Ltd.[15]. The application requires:

1. An accurately defined sensitive volume (region of charge collection). Minimization of charge collection complexity in particular diffusion and funneling effects.

2. An array of identical diodes to improve collection statistics.

3. Diode structure sizes of the order of a few microns to simulate biological cells with typical cell diameters of 10 $15 \mu \mathrm{m}$.

4. Capable of measuring an LET down to about $1 \mathrm{keV} / \mu \mathrm{m}$. Above this value of LET the radiobiological effectiveness begins to rise above 1 .

An ideal silicon based microdosimeter collects charge in a reverse-biased $\mathrm{p}-\mathrm{n}$ junction diode via drift driven separation of electron hole pairs in the depletion region. In reality, charge collection is complicated by additional charge collection via diffusion from outside of the depletion region. By using SOI'structures, we preclude any charge collection from beneath the SOI layer due to the underlying insulating layer of $\mathrm{SiO}_{2}$. Such a design creates a sensitive volume of well-defined depth.

The SOI diode test structures were fabricated on bonded SOI wafers with thickness 2,5 , and $10 \mu \mathrm{m}$. In addition, a bulk device (no SOI) was fabricated. Several diode array structures are available on each device. For this study, the 10 $\mu \mathrm{m}$ SOI device was used to provide a large sensitive volume and best possible signal to noise ratio. The array consisted of $120 \times 40=4800$ diodes with each diode having a junction size of $10 \times 10 \mu \mathrm{m}$. The total size of each diode cell was $30 \times 30 \mu \mathrm{m}$ giving an array area of $0.044 \mathrm{~cm}^{2}$. Note that all diodes in a given array are connected in parallel. The $\mathrm{n}+$ and $\mathrm{p}+$ silicon layers with depths 0.2 and $0.5 \mu \mathrm{m}$ were constructed by arsenic and boron implantations at $30 \mathrm{keV}$ and $5 \times 10^{15} \mathrm{~cm}^{-2}$. The impurity concentration of the $\mathrm{P}$ type silicon was $1.5 \times 10^{15} \mathrm{~cm}^{-3}$. The device is packaged in a 28 Pin DIL ceramic carrier. Figure 1 shows the over-layer and detailed device cross-section whilst Figure 2 is a SEM photograph of a portion of the small array.

The sensitive volume of the device and its radiation hardness were determined by alpha spectroscopy and ionmicrobeam experiments using methods outlined in previous work by us [1]. The device bias was set to $10 \mathrm{~V}$ so that the depletion depth is approximately $3 \mu \mathrm{m}$. Hence the $10 \mu \mathrm{m}$ SOI is not fully depleted and charge collection occurs via diffusion as well as drift processes. For the $10 \mu \mathrm{m}$ device, the charge collection efficiency was found to vary from about 0.79 in regions most distant from a diode junction to 0.86 in the center of a junction where drift collection is strongest. This relatively small variation is a result of the large minority carrier diffusion length $(61 \mu \mathrm{m})$ in comparison to the cell dimensions $(30 \times 30 \times 10 \mu \mathrm{m})$. Therefore, the collection efficiency may be reasonably approximated by a constant value of 0.82 .

Microdosimetric spectra require an estimate of the detectors mean chord length $\bar{l}$. An accurate value of $\bar{l}$ may be obtained via Monte-Carlo simulation methods which transport the protons and other charged particles through the experimental setup and determine the path length traversed through the device. However, a reasonable first order approximation assumes that the beam is largely normal to the diode surface in which case we may approximate $\bar{l} \sim 8.2 \mu \mathrm{m}$.

Two different probe assemblies are used in this work corresponding to different stages in the project development:

Prototype assembly \#1: This probe was the first assembly constructed and consists of the microdosimeter package inserted into a machined Lucite holder with a $1 \mathrm{~m}$ coaxial cable connecting the detector to a Canberra 2003T charge sensitive preamplifier (CSA) as shown in Figure 3. Noise was minimized by encapsulating the entire microdosimeter probe in an aluminum foil shielding which was then connected to the coaxial cable ground. This also 
ensured that the chip did not receive any light, which generates significant noise via surface photo-generation.

An 8-meter coaxial cable links the preamplifier to a Canberra 2024 Fast Spectroscopy amplifier. The output of this amplifier is a voltage pulse whose amplitude is proportional to the energy deposited in the sensitive volume of the microdosimeter. Note that the microdosimeter collects charge generated by the traversal of an ion through the device's sensitive volume. The charge collected is proportional to the energy deposited, as it requires $3.6 \mathrm{eV}$ to generate an electron hole pair in silicon. The pulse height from each event is digitized and stored using a PC based Multi-Channel Analyzer (MCA).

A two-point calibration giving the gain and offset of this system was performed using a polonium-210 alpha spectroscopy source with alpha peak energy of $5.301 \mathrm{MeV}$ and an Americium-241 alpha-gamma source. The $60 \mathrm{keV}$ gamma peak of the Am-241 source was used as a low energy reference point to ensure a good estimation of the calibration offset. The diode array was replaced by a silicon ion implanted detector of similar capacitance, which collects charge corresponding to the total energy of the alpha particle.

Unlike a proportional counter, the silicon diode array does not require a high voltage supply. The supply voltage used in these experiments was $10 \mathrm{~V}$ reverse bias.

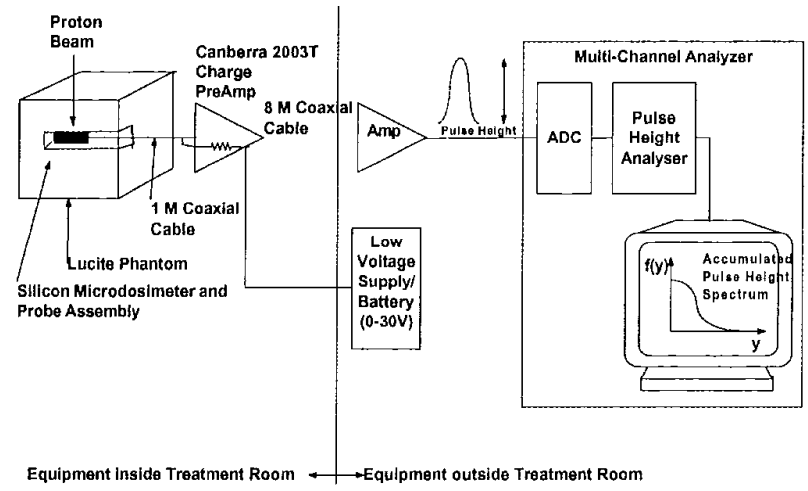

Figure 3: Experimental setup for microdosimetry measurements at PMRC (Tsukuba) using prototype assembly \#1 (original assembly)

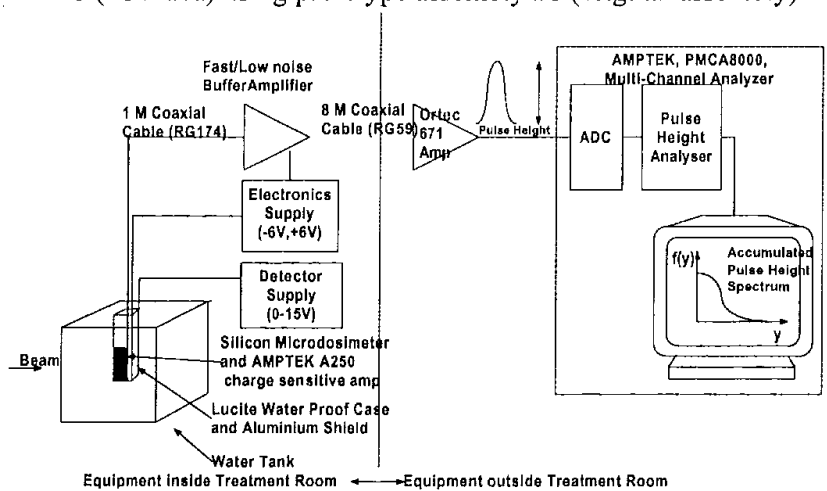

Figure 4: Experimental setup for microdosimetry measurements at NPTC (Boston) using prototype assembly \#2 (low noise)
Prototype assembly \#2: The noise performance of the first prototype could be significantly improved by removing the $100 \mathrm{pF}$ capacitance added by the detector to CSA cable and improving shielding to gain immunity to external electromagnetic fields (particularly RF interference). The second prototype assembly, based on a printed circuit board design, uses an AMPTEK A250 radiation-hard CSA in close proximity to the detector. All components were selected specifically to minimize noise based on our detector characteristics. A system view of the low noise prototype is shown in Figure 4.

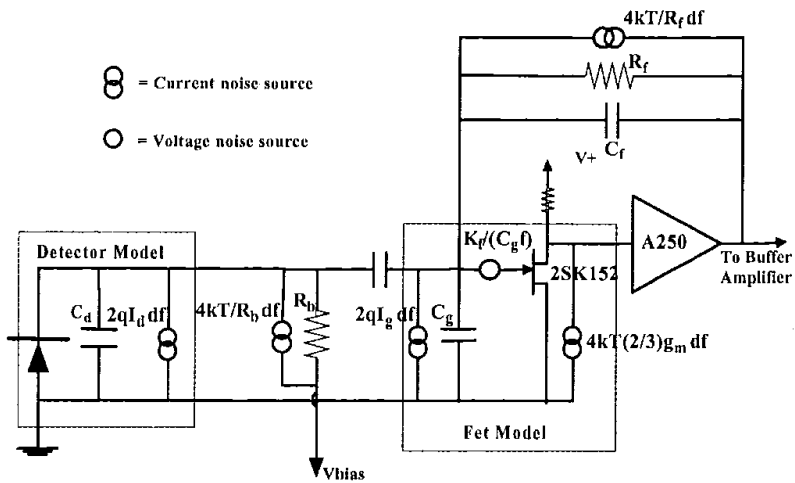

Figure 5: Noise model of microdosimeter and charge sensitive preamplifier

In order to optimize the noise performance of the detector a noise model of the system was developed as depicted in Figure 5. The model considers the primary noise sources as the bias resistor $R_{b}$ and feedback resistor $R_{f}$ thermal noise; the detector current $I_{d}$ shot noise; the thermal noise associated with the input transistor channel resistance and the $1 / \mathrm{f}$ noise of the JFET.

Following standard noise analysis methods such as presented by Delaney and Finch [16] or Radeka [17] we arrive at the following expression for the Equivalent Noise Charge (ENC)

$$
E N C=E \sqrt{\frac{k T \tau}{2 R_{T}}+\frac{q I_{d} \tau}{4}+\frac{K_{f}\left(C_{i n}+C_{f}\right)^{2}}{2 C_{g}}+\frac{k T\left(C_{i n}+C_{f}\right)^{2}}{3 g_{m} \tau}}
$$

where $1 / R_{T}=1 / R_{b}+1 / R_{f}$ and $C_{i n}=C_{d}+C_{g}+C_{\text {stray }}, C_{d}=$ detector capacitance, $\mathrm{C}_{\mathrm{g}}=$ JFET gate capacitance, $\mathrm{C}_{\text {stray }}=$ stray input capacitance, $\tau=$ shaping time constant of spectroscopy amplifier, $\mathrm{K}_{\mathrm{f}}=1 / \mathrm{f}$ noise coefficient, $\mathrm{k}=$ Boltzmann's constant and $\mathrm{T}=$ temperature $(300 \mathrm{~K})$.

The calculation assumes that the feedback time constant $\left(\mathrm{R}_{\mathrm{f}} \mathrm{C}_{\mathrm{f}}\right)$ and input time constant $\left(\mathrm{R}_{\mathrm{b}} \mathrm{C}_{\mathrm{in}}\right)$ are much greater than $\tau$ and that the buffer and spectroscopy amplifier contribute negligible noise. The spectroscopy amplifier frequency response is modeled as a simple $\mathrm{RC}-\mathrm{CR}$ filter

$\frac{V_{\text {out }}}{V_{\text {in }}}=\operatorname{Gain} \frac{\omega \tau}{1+\omega^{2} \tau^{2}}$ 
Typical values for these components and the relative noise contributions are given in Table 1. Therefore minimizing the ENC requires selecting a high $g_{m}$ FET with small gate capacitance and gate current and maximizing $R_{b}$ and $R_{f}$. We select a bias voltage of between 10 and $20 \mathrm{~V}$ since in this range the capacitance is reduced without excessive reverse bias current. The 2SK152 FET was selected for its excellent $\mathrm{g}_{\mathrm{m}} / \mathrm{C}_{\mathrm{g}}$ ratio. Due to the presence of high count rates the shaping time was normally set to $0.25 \mu \mathrm{s}$.

Actual values of noise measured using an Ortec 414 pulsar are $4.7 \mathrm{keV}$ compared with the theoretical estimation of 4.44 $\mathrm{keV}$. The difference may be attributable to additional leakage current on the PCB board, variation in transistor specifications and imperfect shielding of the detector from external noise sources. Given that lower level discriminator settings are typically twice the FWHM we may expect to be able to measure down to around $1.1 \mathrm{keV} / \mu \mathrm{m}$ with this device. The optimum value of noise would involve paralleling several FETs such that the detector and input capacitance matched and also adjusting the shaping time to $0.5 \mu \mathrm{s}$. A noise level of around 300 e rms or $2.5 \mathrm{keV}$ could then be obtained.

Calibration of this low noise prototype is performed as per the older prototype discussed earlier.

Table 1.

Relative contribution of noise components in microdosimeter design

\begin{tabular}{|l|l|l|}
\hline $\begin{array}{l}\text { Noise } \\
\text { Source }\end{array}$ & Relevant Values & $\begin{array}{l}\text { Noise }(\tau=0.25 \mu \mathrm{s}) \\
\text { Electrons (RMS) }\end{array}$ \\
\hline $\mathrm{R}_{\mathrm{b}}$ & $100 \mathrm{M} \Omega$ & 77 \\
\hline $\mathrm{R}_{\mathrm{f}}$ & $300 \mathrm{M} \Omega$ & 45 \\
\hline $\mathrm{I}_{\mathrm{d}}$ & $5 \mathrm{nA}$ & 240 \\
\hline $\begin{array}{l}\mathrm{JFET} \\
(2 \mathrm{SK} 152)\end{array}$ & $\mathrm{K}=10^{-27} \mathrm{~J}, \quad \mathrm{Cg}=6 \mathrm{pF}$, & $\begin{array}{l}254 \text { (channel) } \\
\mathrm{g}_{\mathrm{m}}=20 \mathrm{mS}\end{array}$ \\
\hline $\begin{array}{l}\text { Other } \\
\text { parameters }\end{array}$ & $\begin{array}{l}\tau=0.25 \mu \mathrm{s}, \\
\mathrm{C}_{\mathrm{d}}=45 \mathrm{pF}, \mathrm{C}_{\mathrm{stray}}=5 \mathrm{pF}\end{array}$ \\
\hline Total & & - \\
\hline
\end{tabular}

\section{EXPERIMENT AT PMRC-TSUKUBA}

\section{A. Proton Therapy Facility}

The proton therapy facility at PMRC uses the KEK 500 $\mathrm{MeV}$ booster synchrotron as a beam source [18]. This source was originally designed for HEP experiments and since the energy and intensity are too high for medical applications, the beam energy is degraded down to $250 \mathrm{MeV}$ using carbon based degraders. Additional filters reduced this to $200 \mathrm{MeV}$ for these experiments. In contrast to the NPTC facility, this synchrotron produces $50 \mathrm{~ns}$ pulses with an initial $2 \times 10^{9}$ protons per pulse. The time interval between pulses is about 1 second in parasitic beam mode and 0.05 seconds in full beam mode. Note that the protons/pulse is the same for both parasitic mode and full beam mode. The full beam mode is used for patient treatment and provides a dose rate of approximately $200 \mathrm{cGy} / \mathrm{min}$.

The initial beam intensity is reduced by the carbon degrader followed by a collimator such that around $10^{4}$ protons/pulse are transported to the medical beam line. Then the beam is momentum filtered and shaped for a $10 \times 10 \mathrm{~cm}^{2}$ field. Thus, the beam intensity is around 4 proton/pulse/device which is much higher than at NPTC. This beam presents a difficult experimental challenge even when using the small area of the silicon based microdosimeter. Clearly, the high luminosity of this beam prevents the use of a proportional gas counter for microdosimetry due to strong pile up effects. Note that reducing the beam current does not reduce pile up effects since it usually involves a reduction in the frequency of pulses rather than the number of protons per pulse.

However, to reduce the protons per pulse one can control the momentum slit width $(\mathrm{X})$ and vertical slit width $(\mathrm{Y})$ which are typically set to 150 and $30 \mathrm{~mm}$ respectively.

\section{B. Experimental Setup}

The microdosimeter was placed in a $15 \times 15 \times 30 \mathrm{~cm}^{3}$ Lucite phantom constructed of $2.5 \mathrm{~cm}$ blocks. Such a construction allowed the placement of the detector at different positions along the beam line axis. To avoid pile-up and ensure a constant energy the momentum slit was fixed at $X=3 \mathrm{~mm}$ whilst the vertical slit was varied from $Y=30$ to $2 \mathrm{~mm}$. Microdosimetric spectra at a depth of $17.5 \mathrm{~cm}$ were recorded at various values of vertical slit width to determine the settings required to remove pile-up effects. The acquisition time for the measurements was 600 seconds. Measurements at various depths in the Lucite phantom were then performed having found the beam setup which eliminated pile-up. The integral depth-dose was measured by a MOSFET detector.

\section{Results: Microdosimetric Spectra}

The depth-dose curve obtained using a MOSFET detector placed at various depths in the Lucite phantom are shown in Figure 6. The advantageous characteristic of a MOSFET dosimeter is the extremely small dosimetric volume $(\sim 1 \mu \mathrm{m})$ defined by the gate oxide thickness [19, 20]. High spatial resolution measurements are achievable in the Bragg peak regions. However, the response of the MOSFET is dependent on the particle LET and angle with respect to the oxide electric field. Further investigation and comparison studies should be performed to compare the depth-dose derived from ionization chambers versus MOSFETs.

The microdosimetric spectra obtained as a function of depth in Lucite are shown in Figure 7 . The small peaks from $30-100 \mathrm{keV} / \mathrm{\mu m}$ are due to statistical uncertainties. The general shift in the spectrum to higher lineal energies as depth increases from $14 \mathrm{~cm}$ to $17.5 \mathrm{~cm}$ is evident. The MOSFET depth-dose data indicates that at around $17.5 \mathrm{~cm}$ many of the protons will be approaching the end of their range. In this region, the protons have a higher LET, and therefore higher lineal energy, as they approach the Bragg peak. This behavior is in agreement with results obtained at NPTC and by Coutrakon [11] using a proportional counter.

The data obtained using the prototype assembly \#1 exhibited significant environmental noise problems 
particularly when the beam RF was turned on. It is expected that the second prototype with its significantly improved shielding will avoid such difficulties. The lineal energy could not be obtained below $8 \mathrm{keV} / \mu \mathrm{m}$ due to the high noise levels. This was the motivation for development of the low noise prototype. Despite the limited clinical utility of the incomplete spectrum, we may still verify the capability of the device in terms of high count-rate performance.

The vertical slit was adjusted down to $2 \mathrm{~mm}$ from the normal value of $30 \mathrm{~mm}$ in order to avoid pile-up. Figure 8 shows a comparison of microdosimetric spectrum obtained under Parasitic-Beam and Full-Beam modes at PMRC with different vertical slit widths (depth in Lucite phantom $=17.9$ $\mathrm{cm}$ ). This example shows significant pile-up effects using the Parasitic Beam despite its much lower total intensity. To avoid pile-up in either mode the beam requires a vertical slit width of $2 \mathrm{~mm}$ in order to reduce the number of protons/spill. The spectra of Figure 7 obtained at various depths used such a slit arrangement. The results indicate that the low noise prototype applied to similar beam conditions should be capable of measuring the microdosimetric spectrum down to $1.2 \mathrm{keV} / \mu \mathrm{m}$ without significant pile-up complications.

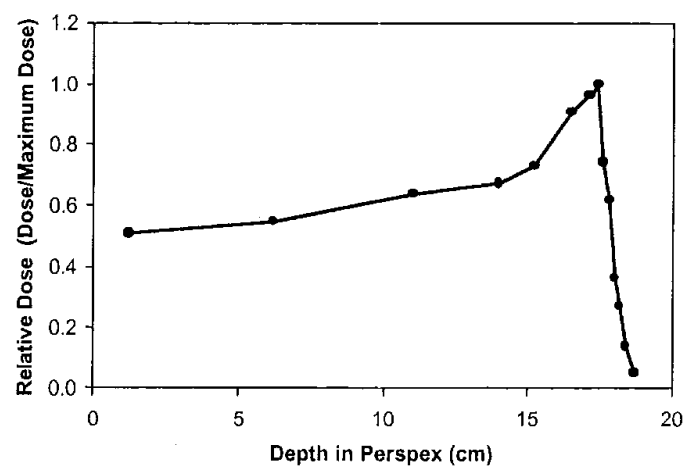

Figure 6: Bragg peak of $200 \mathrm{MeV}$ proton beam (PMRC-Tsukuba) derived by MOSFET detector in Lucite phantom. Relative dose is the dose normalized to maximum dose at depth of $17.4 \mathrm{~cm}$.

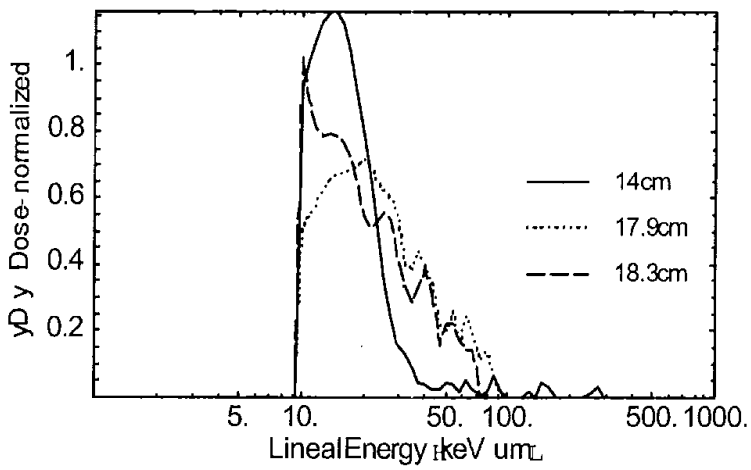

Figure 7: Microdosimetric spectra of $200 \mathrm{MeV}$ proton beam (PMRCTsukuba) at various depths in a Lucite phantom. Range of proton is approximately $18 \mathrm{~cm}$.

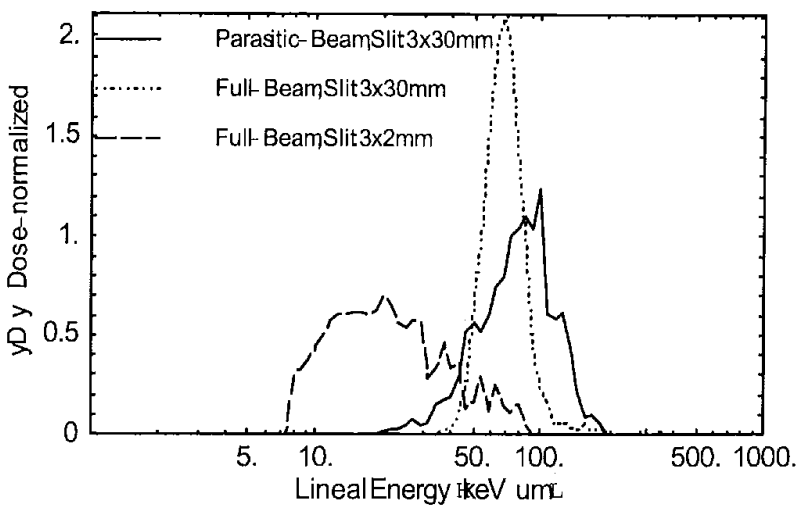

Figure 8: Comparison of microdosimetric spectrum obtained under Parasitic-Beam and Full-Bcam modes at PMRC-Tsukuba with different vertical slit widths (depth in Lucite phantom $=17.9 \mathrm{~cm}$ ). This example shows significant pile-up effects using the Parasitic Beam despite its much lower total intensity. To avoid pile-up in either mode the beam requires a vertical slit width of $2 \mathrm{~mm}$ in order to reduce the number of protons/spill.

\section{EXPERIMENT AT NPTC-BOSTON}

\section{A. Proton Therapy Facility}

The new proton therapy facility at NPTC utilizes a 230 $\mathrm{MeV}$ beam extracted from an isochronous cyclotron [21, 22]. The accelerator was built by Ion Beam Applications (IBA), Belgium and has a maximum current of $1.5 \mu \mathrm{A}$ which is hardware limited to $300 \mathrm{nA}$ in order to limit the maximum dose rate to the patient. After extraction from the cyclotron the beam passes through the Energy Selection System (ESS) designed to give a beam of any energy between 70 and 230 $\mathrm{MeV}$ with a user defined momentum width and emittance. The ESS uses a rapidly adjustable rotating variable thickness of graphite. Slits are provided just after the energy degrader to reduce the beam divergence to $\Delta \mathrm{E} / \mathrm{E}< \pm 1 \%$. The facility uses a range modulator filter to create the desired spread in the proton Bragg peak. A double scattering system using a fixed scatterer and a second non-uniform thickness scatterer produce a beam with uniform intensity in the lateral profile over a $10 \times 10 \mathrm{~cm}^{2}$ field size.

The NPTC cyclotron operates in continuous wave mode using a $106 \mathrm{MHz} \mathrm{RF}$ frequency. Acceleration of protons occurs within 10-15 degrees of the RF wave. The time structure of the beam is therefore semi-DC with a duty-cycle of about $10 \%$ (30deg $330 \mathrm{deg}$ ). A typical treatment beam is 2 $\mathrm{nA}$ equivalent to $1.25 \times 10^{10}$ protons/second. So in a single RF cycle of 9.4 ns there are 118 protons spread over $0.8 \mathrm{~ns}$. For the case of a $10 \times 10 \mathrm{~cm}^{2}$ field size (obtained using a double scattering method) we can expect $1.2 \mathrm{p} / \mathrm{cm}^{2} / \mathrm{cycle}$. Under these conditions, a microdosimeter should have a small cross-sectional area and fast collection time. 


\section{B. Experimental Setup}

The microdosimeter selected has an active cross-sectional area of $0.044 \mathrm{~cm}^{2}$. The minimum amplifier shaping time available was $0.25 \mu \mathrm{s}$ and the Ortec pile-up reject system was employed to eliminate pile-up signals. Note that decreasing the amplifier shaping time further would increase noise to unacceptable levels. In order to maintain a reasonable deadtime (less than about $20 \%$ ) we require less than 1 proton per $1.25 \mu$ s striking the detector. Such a requirement may be fulfilled using a beam current of $0.25 \mathrm{nA}$ in which case we expect $0.7 \mathrm{p} / \mu \mathrm{s} /($ device area). In practice, higher beam currents (up to $1 \mathrm{nA}$ ) could be employed without excessive dead-time and distortion of the spectra due to pile-up events. The quick calculations performed above do not include losses of beam current due to scattering outside of the beam field.

The microdosimeter was placed in a Scandtronix water tank (Model RFA-300 Plus) which enabled rapid remote adjustment of the detector position. The detector position was aligned with the center of the beam and microdosimetric spectra were recorded at various distances from the beam side wall of the tank. At each depth, spectra were recorded using two different gains to provide adequate dynamic range in the final merged spectrum. Initially the beam current was adjusted to ensure that the spectra were not corrupted by pileup events. Furthermore, during measurements, the signal prior to the MCA was monitored continuously with an oscilloscope to ensure the average interval between pulses was reasonable and no pileup was present. The typical acquisition time was 600 seconds.

In addition, measurements of the depth-dose profile in the water tank were performed using a Markus ionization chamber. Measurements were made every $0.3 \mathrm{~cm}$ for the range $0-30 \mathrm{~cm}$ along the beam axis.

The proton beam was setup with the energy degrader in pass though position and the ESS configured to provide the full cyclotron energy of $230.5 \mathrm{MeV}$ with an $\Delta \mathrm{E} / \mathrm{E}$ of $0.18 \%$. The cyclotron energy is attenuated by the items identified in Table 2 so that the final energy (calculated using SRIM code [23]) of protons entering the water is $191.5 \mathrm{MeV}$.

Table 2.

Beam items in nozzle of NPTC

\begin{tabular}{|l|l|l|l|l|}
\hline Item & Material & $\begin{array}{l}\text { Thick } \\
(\mathrm{cm})\end{array}$ & $\begin{array}{l}\text { Eout } \\
(\mathrm{MeV} \\
)\end{array}$ & $\begin{array}{l}\text { Elost } \\
(\mathrm{MeV} \\
)\end{array}$ \\
\hline Cyclotron & - & - & 230.5 & - \\
\hline Fixed scatterer & $\mathrm{Pb}$ & 0.131 & 227.3 & 3.2 \\
\hline Range Mod. 1 & $\mathrm{Pb}$ & 0.4254 & 216.7 & 10.6 \\
\hline Range Mod. 2 & $\mathrm{C}$ & 0.0773 & 216.0 & 0.7 \\
\hline Beam Spread & Water Eq & 3.65 & 199.8 & 16.2 \\
\hline Nozzle air & Air & 285 & 198.3 & 1.4 \\
\hline Ion chambers & Water Eq & 0.304 & 197.0 & 1.4 \\
\hline Phantom wall & Lucite & 1 & 191.5 & 5.5 \\
\hline
\end{tabular}

Water Eq: means that the effective thickness is specified in water equivalent terms. The beam spreading filter is made of lead and Lexan.

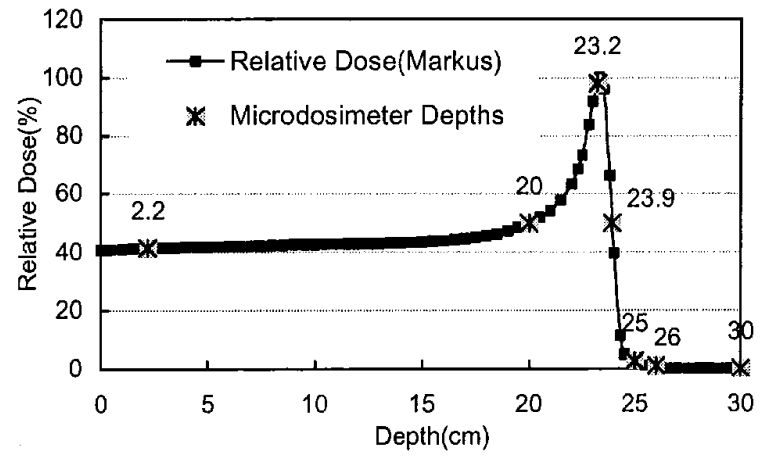

Figure 9: Depth-Dose curve for the NPTC $191.5 \mathrm{MeV}$ proton beam in water. Relative dose is defined as Dose(Markus)/Maximum Dose(Markus). The maximum dose is at $23.2 \mathrm{~cm}$ and the relative dose $=1$ at this point. The depths at which microdosimetric measurements are made are shown.

\section{Results: Microdosimetric Spectra}

The microdosimetric spectra obtained as a function of depth at NPTC are shown in Figure 10. One first notes the significantly improved noise performance of the low noise prototype in comparison to measurements made at PMRC. Measurements were obtained down to the noise level of about $1.2 \mathrm{keV} / \mu \mathrm{m}$. The depth-dose curve obtained using the Markus chamber and the microdosimeter measurement positions are shown in Figure 9. At depths less than $20 \mathrm{~cm}$, the large dose contribution from low lineal energy events is due to significant high energy proton and gamma contributions which both have an LET below $2 \mathrm{keV} / \mu \mathrm{m}$. As we approach the Bragg peak, the LET of the protons rises as seen by the increase in the mean lineal energy and the shift of the spectra to higher lineal energies at depths between $20-26 \mathrm{~cm}$. These effects were also observed using a proportional counter at the Loma Linda proton beam by Coutrakon et.al. [11] This shift is not due to pileup effects since, firstly, the signal was continuously monitored with an oscilloscope and, secondly, the count rate is lower at higher depths due to beam spreading.

Note the step decline in the microdosimetric spectra at around the maximum LET of protons of $78 \mathrm{keV} / \mu \mathrm{m}$. Dose contributions are evident at lineal energies above the maximum proton LET due to nuclear reaction products such as alpha particles and heavy ion recoils. However, the relative number of such events is much smaller than the proton events hence the step change above the maximum proton LET. As we exceed the maximum range of the protons, the higher lineal energy components contribute a relatively higher proportion of dose. At $30 \mathrm{~cm}$, well past the primary proton range, the spectrum is comprised totally of neutron reaction products including recoil protons, alpha particles and heavy ion recoils. The neutron reaction products tend to have a large proportion of dose at higher lineal energies. 


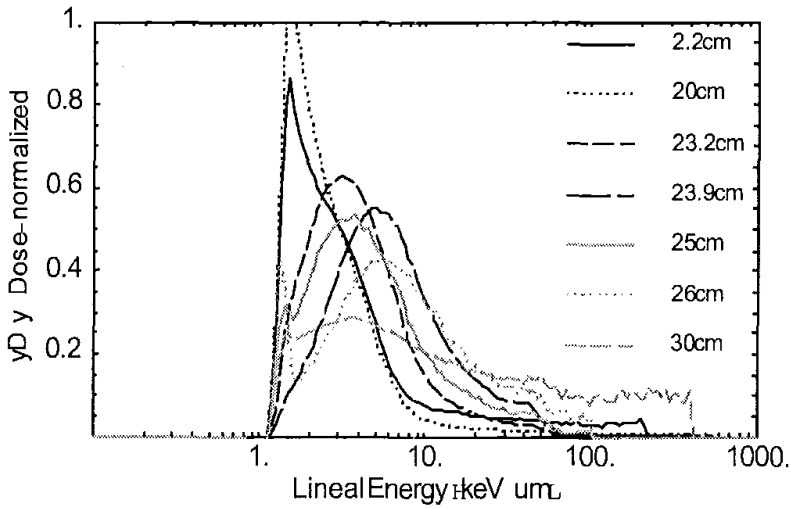

Figure 10: Microdosimetric spectra of $191.5 \mathrm{MeV}$ proton beam at various depths in a water phantom. Range of proton is approximately $23.7 \mathrm{~cm}$.

\section{CONCLUUSION}

These measurements provide valuable information for radiobiological studies of the varying effectiveness of the beam as a function of depth. Future work may be directed towards the analysis of beam relative biological effectiveness such as performed at Loma Linda by Coutrakon et. al [11] at a higher spatial resolution than previously attempted.

In general, pile up and perturbation effects were avoided due to the small area $\left(0.04 \mathrm{~cm}^{2}\right)$ of the total pixel array. Pileup did occur for experiments at PMRC when the vertical momentum slit width was greater than $2 \mathrm{~mm}$. Under these conditions, an even smaller area detector is required. The detector was coupled to a radiation-hard charge sensitive amplifier with the probe assembly capable of measuring an LET down to $1.2 \mathrm{keV} / \mu \mathrm{m}$. The device offers much improved spatial resolution compared with a proportional gas counter particularly in the critical high dose region around the proton Bragg peak.

We have demonstrated a new approach to microdosimetry in radiation oncology using a silicon SOI p-n junction array applied to proton therapy beams. Low noise, small size and good radiation hardness facilitate the application of this device in radiation oncology.

\section{ACKNOWLEDGEMENTS}

The authors would like to thank Shigeo Satoh of Fujitsu Research Laboratories for providing the diode array samples and the staff of PMRC at Tsukuba and NPTC at Boston for their generous time and effort. Electronics support at Boston was supplied by J. Burns and R.M. Cataldo whilst at NPTC Y. Kohno greatly assisted experiments. Appreciation is extended to Jacquelyn Yanch (MIT) who was extremely supportive in our trip to Boston. We are also indebted to John Burke (University of Wollongong) for his outstanding skills in manufacturing the Lucite probes and James Ziegler (IBM) for the latest copy of SRIM. This work was funded by Australian National Health and Medical Research Council
Grant No 960804 and an Australian Department of Industry, Science and Technology grant.

\section{REFERENCES}

[1] P. D. Bradley, A. B. Rosenfeld, K. K. Lee, D. Jamieson, G. Heiser, and S. Satoh, "Charge collection and radiation hardness of a SOI microdosimeter for space and medical applications," IEEE Trans. Nucl. Sci, vol. 45, no. 6, pp. 2700-2710, 1998.

[2] P. D. Bradley, A. B. Rosenfeld, B. J. Allen, J. Corderre, and J. Capela, "Performance of silicon microdosimetry detectors in boron neutron capture therapy," Radiat. Res., vol. 151, pp. 235-243, 1999.

[3] P. D. Bradley, A. B. Rosenfeld, B. J. Allen, T. Kobayashi, Y. Sakurai, C.Kota, R. L. Maughan, M.Yudelev, J. Coderre, and J. Capala, "Application of silicon diode arrays for microdosimetry in BNCT and FNT," 8th International Symposium on Neutron Capture Therapy for Cancer, La Jolla, USA, 14-18th Sep 1998, 1998.

[4] D. W. Miller, "A review of proton beam radiation therapy," Med. Phys., vol. 22, no. 11, pp. 1943-1954, 1995.

[5] International Commission on Radiation Units and Measurements, Stopping powers for protons and alpha particles (ICRU report 49). Bethesda, Md., U.S.A.: ICRU, 1993.

[6] S. M. Seltzer, An assessment of the role of charged secondaries from nonelastic nuclear interactions by proton therapy beams in water. Gaithersberg,MD: U.S Dept of Commerce, 1993.

[7] P. J. Kliauga, R. D. Colvett, Y. L. Lam, and H. H. Rossi, "The relative effectiveness of $160 \mathrm{MeV}$ protons. I. Microdosimetry"," International Journal of Radiation Oncology, Biology, and Physics, vol. 4, pp. 1001-1008, 1978.

[8] J. B. Robertson, J. M. Eaddy, J. O. Archambeau, G. B. Coutrakon, D. W. Miller, M. F. Moyers, J. V. Siebers, J. M. Slater, and J. F. Dicello, "Relative biological effectiveness and microdosimetry of a mixed energy field of protons up to $200 \mathrm{MeV}$," Adv. Space Res., vol. 14, pp. 271-275, 1994.

[9] V. P. Cosgrove, A. C. Aro, S. Green, M. C. Scott, G. C. Taylor, D. E. Bonnett, and A. Kacperek, "Studies relating to $62 \mathrm{MeV}$ proton cancer therapy of the eye," Radiation Protection Dosimetry, vol. 44, pp. 405-409, 1990.

[10] V. P. Cosgrove, S. Delacroix, S. Green, A. Mazal, and M. C. Scott, "Microdosimetric studies on the Orsay proton synchrocyclotron at 73 and $200 \mathrm{MeV}$," Radiation Protection Dosimetry, vol. 70, no. 1-4, pp. 493-496, 1997. 
[11]G. Coutrakon, J. Cortese, A. Ghebremedhin, J. Hubbard, J. Johanning, P. Koss, G. Maudsley, C. R. Slater, C. Zuccarelli, and J. Robertson, "Microdosimetry spectra of the Loma Linda proton beam and relative biological effectiveness comparisons," Med. Phys., vol. 24, no. 9, pp. 1499-1506, 1997.

[12]R. Becker, J. Bienen, U. Carl, and P. Cloth, "Biophysical investigations of therapeutic proton beams," Radiation Protection Dosimetry, vol. 70, no. 14, pp. 485-492, 1997.

[13]H. H. Rossi and M. Zaider, Microdosimetry and Its Applications. London: Springer, 1996.

[14]P. D. Bradley and A. B. Rosenfeld, "Tissue Equivalence Correction for Silicon Microdosimetry Detectors in Boron Neutron Capture Therapy," Med. Phys., vol. 25, no. 11, pp. 2220-2225, 1998.

[15] Y. Tosaka, S. Satoh, K. Suzuki, T. Sugii, N. Nakayama, H. Ehara, G. A. Woffinden, and S. A. Wender, "Measurements and Analysis of Neutron-ReactionInduced Charges in a Silicon Surface Region," IEEE Trans. Nucl. Sci., vol. 44, no. 2, pp. 173-178, 1997.

[16]C. F. G. Delaney and E. C. Finch, Radiation Detectors: Physical Principles and Applications, 1 ed. Oxford: Clarendon Press, 1992.

[17] V. Radeka, "Low-Noise Techniques in Detectors," Ann. Rev. Nucl. Part. Sci., vol. 38, pp. 217-277, 1988.

[18]T. Matsuda and K. Inamura, "Computer controlled multi-leaf conformation radiotherapy," Nippon Act. Radiol, vol. 41, pp. 965-974, 1981.

[19] A. B. Rosenfeld, M. G. Carolan, G. I. Kaplan, B. J. Allen, and V. I. Khivrich, "MOSFET dosimeters: The role of encapsulation on dosimteric characteristics in mixed gamma-neutron and megavoltage X-ray fields.," IEEE Trans. Nucl. Sci., vol. 42, no. 6, pp. 1870-1877, 1996.

[20] A. B. Rosenfeld, G. I. Kaplan, M. G. Carolan, B. J. Allen, R. Maughan, M. Yudelev, C. Kota, and J. Coderre, "Simultaneous macro-micro dosimetry with MOSFETs," IEEE Trans. Nucl. Sci., vol. 43, no. 6, pp. 2693-2700, 1996.

[21] A. Smith, M. Goitein, H. Suit, S. Durlacher, J. Flanz, K. Gall, A. Levine, S. Rosenthal, and S. Woods, "The Massachusetts General Hospital Northeast Proton Therapy Center, Boston Massachusetts," presented at Proceedings of NIRS International Seminar on the application of heavy ion accelerator to radiation therapy of cancer in connection with XXI PTCOG meeting, Chiba, Japan, 1994.

[22] J. Sisterton, J. B. Flanz, and J. E. Burns, "A new proton irradiation facility at the Northeastern Proton Therapy Center," , IEEE Trans. Nucl. Sci., vol. 45, no. 6, 1999.

[23] J. F. Ziegler, "SRIM: The Stopping and Range of Ions in Matter,", 97.02 ed. Yorktown: IBM Research, 1997. 